\title{
A parental requirement for dual-specificity phosphatase 6 in zebrafish
}

\author{
Jennifer M. Maurer and Charles G. Sagerström
}

\begin{abstract}
Background: Signaling cascades, such as the extracellular signal-regulated kinase (ERK) pathway, play vital roles in early vertebrate development. Signals through these pathways are initiated by a growth factor or hormone, are transduced through a kinase cascade, and result in the expression of specific downstream genes that promote cellular proliferation, growth, or differentiation. Tight regulation of these signals is provided by positive or negative modulators at varying levels in the pathway, and is required for proper development and function. Two members of the dual-specificity phosphatase (Dusp) family, dusp6 and dusp2, are believed to be negative regulators of the ERK pathway and are expressed in both embryonic and adult zebrafish, but their specific roles in embryogenesis remain to be fully understood.
\end{abstract}

Results: Using CRISPR/Cas9 genome editing technology, we generated zebrafish lines harboring germ line deletions in dusp6 and dusp2. We do not detect any overt defects in dusp2 mutants, but we find that approximately $50 \%$ of offspring from homozygous dusp6 mutants do not proceed through embryonic development. These embryos are fertilized, but are unable to proceed past the first zygotic mitosis and stall at the 1-cell stage for several hours before dying by $10 \mathrm{~h}$ post fertilization. We demonstrate that dusp6 is expressed in gonads of both male and female zebrafish, suggesting that loss of dusp6 causes defects in germ cell production. Notably, the 50\% of homozygous dusp6 mutants that complete the first cell division appear to progress through embryogenesis normally and give rise to fertile adults.

Conclusions: The fact that offspring of homozygous dusp6 mutants stall prior to activation of the zygotic genome, suggests that loss of dusp6 affects gametogenesis and/or parentally-directed early development. Further, since only approximately $50 \%$ of homozygous dusp6 mutants are affected, we postulate that ERK signaling is tightly regulated and that dusp6 is required to keep ERK signaling within a range that is permissive for proper embryogenesis. Lastly, since dusp6 is expressed throughout zebrafish embryogenesis, but dusp6 mutants do not exhibit defects after the first cell division, it is possible that other regulators of the ERK pathway compensate for loss of dusp6 at later stages.

Keywords: CRISPR, ERK signaling, Dual-specific phosphatase, MAP kinase phosphatase, Germ cell development, Zebrafish embryonic patterning

\section{Background}

The extracellular signal-regulated kinase (ERK) pathway is a major signaling cascade that promotes proliferation and differentiation in many different cell types. As one of the mitogen-activated protein (MAP) kinase pathways, the canonical ERK pathway receives signals from receptors for a growth factor or hormone, such as fibroblast growth factor (FGF), epidermal growth factor (EGF), and plateletderived growth factor (PDGF), which then activates a MAP kinase kinase kinase (Raf), a MAP kinase kinase

\footnotetext{
* Correspondence: charles.sagerstrom@umassmed.edu

Department of Biochemistry and Molecular Pharmacology, University of Massachusetts Medical School, Worcester, MA, USA
}

(MEK), and finally the MAP kinase ERK. Phosphorylated and activated ERK then moves into the cell nucleus, where it can activate transcription factors to initiate target gene expression. During early development, ERK signaling is active in several critical regions of the zebrafish embryo. For example, ERK signaling works cooperatively with Wnt signaling to promote trunk elongation and the formation of somites in the tailbud [1], and triggers the differentiation of lens fiber cells in the developing eye [2]. It has been demonstrated that ERK signaling is required for proper patterning, especially within the hindbrain, where the cascade is initiated by the FGF pathway, defines the forming rhombomere boundaries, and sets up the 
anterior-posterior axis [3-6]. Zebrafish embryos treated with an inhibitor of the FGF receptor upstream of ERK lack the fifth and sixth rhombomere ( $\mathrm{r} 5$ and $\mathrm{r} 6$ ) of the hindbrain and the neurons that normally develop in those regions [5]. Similar to other major signaling pathways, the ERK pathway is able to induce the expression of its own regulators. Many such proteins, including members of the dual-specificity phosphatase (Dusp) and sprouty (Spry) families, Sef, and FLRT, are expressed downstream of the pathway [7]. These proteins interact with upstream pathway components or with ERK itself, and provide positive or negative feedback to modulate the signaling pathway [3, 8-10].

Early embryonic patterning is also driven by the hox genes, a key family of homeodomain-containing transcription factors that control cell fate specification [11, 12]. Notably, a microarrary screen identified a Dusp family member, dusp2 (also called PAC-1 or wu:fj40g04), as a hoxb1b-inducible gene in zebrafish [13]. The Dusp family comprises a group of proteins that remove phosphates from both serine/threonine and tyrosine residues of mitogen-activated protein (MAP) kinases resulting in their inactivation. Previous work has shown that Dusp2 is an inducible, nuclear protein that has a strong specificity for ERK $[9,14-19]$. There is also evidence that Dusp2 is capable of dephosphorylating p38 in vitro [14] and JNK in vivo [15]. In accordance with it being hoxb1b-regulated, dusp 2 is expressed in rhombomere $4(\mathrm{r} 4)$ of the hindbrain - a region that requires hoxb1b function. A very similar protein, Dusp6 (also called MKP3), is expressed in several regions of the early embryo, including in $\mathrm{r} 4$ where its expression overlaps with dusp2 and hoxb1b [20]. In contrast to Dusp2, Dusp6 is a cytoplasmic protein and has confirmed roles in developmental signaling, including axial patterning, limb development, organ size regulation, and somite formation [20-22]. The fact that dusp 2 and dusp6 are co-expressed with hoxb1b in $\mathrm{r} 4$, and that dusp 2 is hoxb1b inducible, suggests a potential role for hox genes in controlling ERK signaling. Loss of function dusp 2 mice were reported to develop normally, but this was not analyzed in detail [15]. Loss of function dusp 6 mice and morphant zebrafish have been analyzed, and the effects in these animals mimic mutations that inappropriately active FGF receptors [20, 21]. However, these phenotypes differ significantly between the two species. Notably, the analysis in zebrafish made use of anti-sense morpholino oligos (MOs), whose reliability has recently been called into question $[23,24]$.

Here we used the CRISPR/Cas9 genome editing system to generate loss of function zebrafish mutants for both dusp 2 and dusp6. We do not detect any developmental defects in dusp 2 mutants, but find that embryos derived from homozygous dusp6 mutant parents have reduced viability. These embryos are unable to undergo the first cell division and stall at the 1-cell stage. Our results indicate that this phenotype is independent of the zygotic genome, and instead suggest a parental requirement for dusp6 in zebrafish embryogenesis.

\section{Methods}

\section{Zebrafish care}

Wildtype Ekkwill and mutant zebrafish lines were raised in the University of Massachusetts Medical School Zebrafish Facility. All embryos were staged according to morphological criteria and hours or minutes post fertilization [25].

\section{Zebrafish embryonic injections}

Embryos were collected from natural matings immediately following fertilization. Collected embryos were aligned on an agarose mold and injected with 1-2 ng of injection mix using a borosil needle, micromanipulator, and dissecting microscope. For the injections of $f g f 8$ mRNA, a plasmid containing the full coding sequence of $f g f 8$ was in vitro transcribed. This mRNA was diluted in water and phenol red to a final concentration of $5-500 \mathrm{ng} / \mu \mathrm{l}$ and injected into 1-cell embryos.

\section{Generation and injection of CRISPR guide RNAs}

CRISPR target sites were selected based on their proximity to the start and stop codons of the coding sequence of the targeted gene, and also by the requirement for a protospacer adjacent motif (PAM) sequence (NGG) at the $3^{\prime}$ end of target site. We created and annealed oligos containing a T7 promoter sequence, the target sequence, and an additional constant region to create the template for the guide RNAs (Additional file 1). These templates were transcribed in vitro using T7 RNA polymerase (Promega) in a reaction containing transcription buffer (Promega), RNase inhibitor (Promega), and rNTPs. A linearized plasmid encoding cas9 [26] was also transcribed in vitro using the Sp6 mMessage mMachine Kit (Ambion). The two guide RNAs targeting each gene were combined with cas 9 mRNA and phenol red, and 1-2 nl of this mixture was injected into the cell of early 1-cell stage embryos.

\section{Identification of germ line mutations and genotyping}

For both dusp 6 and dusp 2 mutants, the embryos injected with the guide RNAs and cas 9 mRNA mixture were raised as the F0 generation. At 3 months of age, these fish were individually crossed to a wildtype fish (Fig. 2c). Half of each resulting clutch was raised to adulthood as the F1 generation. Genomic DNA was extracted from the embryos in the remaining half of the clutch to confirm activity of the guide RNAs. This genomic DNA was screened for deletions by PCR using primers that flank the region between the two guide RNA target sites (Fig. 2a, Additional file 2). Amplification from mutant sequences containing large deletions will produce $400-600$ bp products (Fig. 2b, c, 
Table 1). In contrast, amplification from wildtype sequences will produce products greater than $1 \mathrm{~kb}$ and these fragments may not amplify well under the PCR conditions used. F1 adults derived from positive clutches were individually genotyped with fin clip DNA using the same PCR primers. To confirm that these fish were heterozygous, a second set of primers was used to amplify only the wildtype sequence where one or both primers were placed inside the deletion (Fig. 2a, Additional file 2). F1 heterozygous fish were crossed to generate homozygous mutants.

\section{Anti-sense morpholino oligo knockdowns}

An anti-sense morpholino oligo (MO) was designed to the dusp 6 translation start site with the sequence $5^{\prime}$-T ACCGTGAGACCTTAAAACTGCGGA-3'. A MO targeted to the dusp2 translation start site with the sequence 5' -GTCGCCGATACCCATGATGCCCTCT-3' was also designed. As a control, a 5-mismatch control oligo was designed with the sequence 5 '-GTCcCCcA TAgCCATcATcCCCTCT-3'. All MOs were generated by Gene Tools, LLC and re-suspended in distilled water for a stock solution of $3 \mathrm{mM}$. The stock solution was further diluted with water and phenol red and 1-2 $\mathrm{nl}$ was injected into the yolk of 1-cell stage embryos.

\section{RNA-seq library preparation}

Total RNA was extracted from pools of de-chorionated,

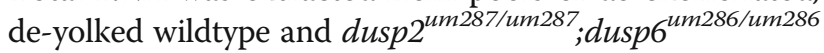
embryos at $18 \mathrm{hpf}$ using the RNeasy Mini Kit (Qiagen). Three libraries from wildtype embryos and three libraries from dusp $2^{u m 287 / u m 287} ;$ dusp $6^{\text {um286/um } 286}$ embryos were then generated from $3 \mu \mathrm{g}$ RNA using the TruSeq Stranded mRNA Library Prep Kit (Illumina). All libraries were analyzed for quality on a bioanalyzer prior to sequencing (Agilent 2100 BioAnalyzer).

\section{Processing and analysis of RNA-seq data}

Fastq files containing strand-specific and filtered reads were processed using the University of Massachusetts Medical School Dolphin web interface [27]. Reads were quality checked using FastQC and aligned to the DanRer7 zebrafish transcriptome using RSEM. After filtering out ribosomal RNA read counts, differentially-expressed genes were identified as those with a greater than 2-fold change in expression between the wildtype and dusp $2^{u m 287 /}$ ${ }^{\text {um } 287} ;$ dusp $6^{\text {um286/um286 }}$ samples.

\section{In situ RNA hybridization, immunostaining, and nuclear staining}

For in situ hybridization, embryos were fixed at the appropriate time point in $4 \%$ paraformaldehyde and stored in $100 \%$ methanol at $-20^{\circ} \mathrm{C}$. RNA hybridization was performed as described and was followed by a color reaction using NBT/BCIP or INT/BCIP in 10\% polyvinyl alcohol [28]. RNA probes for the following genes were produced by cloning a 900-1000 bp fragment of the coding sequence into a vector and transcribing an anti-sense transcript: dusp6, dusp2, krox20, hoxb1a, six7, pea3, erm, fgf3, fgf8, valentino, bmp $2 b$, bmp 4 , chordin, and noggin1. The ot $x 5$ probe was purchased from the Zebrafish International Resource Center.

For whole-mount immunostaining, embryos were fixed in $4 \%$ paraformaldehyde/8\% sucrose/1× PBS. Fluorescent antibody staining was performed as described previously [29]. Commercially-available primary antibodies used: mouse 3A10 (1:100; Developmental Studies Hybridoma Bank [DSHB]), mouse anti-Islet1/2 (39.4D5; 1:100; DSHB), rabbit anti-phospho-p44/42 MAPK ERK1/2 (1:250; Cell Signaling Technology 4370), rabbit antiphospho-histone H3 (1:200; Abcam 5176), mouse RMO44 (1:100; Fisher Scientific 13-0500), and mouse anti-Zn8 (1:1000; DSHB). An antibody against Valentino was generated by immunizing rabbits with a GST-tagged full-length zebrafish Valentino protein. This antibody was purified using an IgG Purification Kit (Dojindo Molecular Technologies) and used at a concentration of 1:100. Secondary antibodies used: goat anti-mouse Alexa Fluor 488 (1:200; Molecular Probes A11001), goat anti-rabbit Alex Fluor 568 (1:200; Molecular Probes A110011), and goat antirabbit IgG-HRP (1:1000; Abcam 6789; detected with PerkinElmer's TSA Plus Fluorescein System).

For nuclear staining, embryos were fixed in $4 \%$ paraformaldehyde and stored in $100 \%$ methanol at $-20^{\circ} \mathrm{C}$. Rehydrated whole embryos were stained with $0.5 \mu \mathrm{g} / \mathrm{ml}$

Table 1 Characteristics of CRISPR guide RNAs targeting dusp6 and dusp2

\begin{tabular}{|c|c|c|c|c|c|}
\hline CRISPR guide & Target coordinate $^{a}$ & Target sequence ${ }^{b}$ & Strand $^{\mathrm{c}}$ & Size of mutant PCR band ${ }^{d}$ & Mutagenesis rate $^{\mathrm{e}}$ \\
\hline dusp6-5' & Chr25:18233489 & GAGCCTCATGCTCCGGCGAC & - & $\sim 564 \mathrm{bp}$ & $2 / 23$ \\
\hline dusp6-3' & Chr25:18231243 & CTCGAGTCCACGTGAGGTCC & - & & \\
\hline dusp2-5' & Chr8:40589831 & GGCGACCCTCTCGAGATCTC & + & $\sim 392 \mathrm{bp}$ & $3 / 23$ \\
\hline dusp2-3' & Chr8:40592681 & ACACTGTGACAGATCTACAA & + & & \\
\hline
\end{tabular}

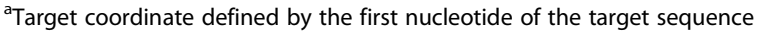

${ }^{\mathrm{b}}$ Genomic sequence targeted by the guide RNA

'Strand of genomic DNA which is targeted by the guide RNA

${ }^{\mathrm{d}} \mathrm{PCR}$ product size if a CRISPR-induced deletion is present (see Additional file 2 for primer sequences)

${ }^{\mathrm{e}}$ The number of FO germ line positive founders identified out of those screened 
DAPI solution in distilled water for $15 \mathrm{~min}$, and then washed for several hours.

For imaging, embryos older than 24hpf were dissected from the yolk and flat-mounted in $70 \%$ glycerol for imaging on bridged coverslips. Images were captured using a Nikon Eclipse E600 microscope equipped with a Nikon 20x Plan Fluor objective and a Zeiss Axiocam 503 color camera. Embryos between 1 and 24hpf were suspended in 3\% methyl cellulose for imaging. Images were captured using a Leica M165 FC microscope equipped with a Leica DFC310 FX camera. Embryos younger than $1 \mathrm{hpf}$ were mounted in $70 \%$ glycerol in depression slides for imaging. All images were imported into Adobe Photoshop and adjustments were limited to contrast, levels, and cropping; all adjustments were applied to the entire image.

\section{Quantitative PCR}

Total RNA was extracted from whole embryos, or from dissected organs of the adult fish, using the RNeasy Mini Kit (Qiagen). At least $100 \mathrm{ng}$ of RNA was used to reverse transcribe cDNA using the High Capacity cDNA Reverse Transcription Kit (Applied Biosystems). The qPCR reaction was carried out using SYBR Green qPCR Master Mix (BioTool) on an Applied Biosystems 7300 PCR System. Results were normalized to those of a housekeeping gene (b-actin or odc1).

\section{Results}

\section{Knockdown of dusp6 and dusp2 via MO results in a hindbrain phenotype}

We initially used anti-sense morpholino oligos (MOs) to assess the function of $d u s p 6$ and $d u s p 2$ by designing MOs to the translation start site to prevent synthesis of Dusp6 and Dusp2 protein (Fig. 1b). Since dusp6 and dusp2 are both expressed in rhombomere $4(\mathrm{r} 4)$ of the hindbrain (Fig. 1a), possibly by acting downstream of hoxb1b [13], we examined hindbrain development in MO-injected embryos. $\mathrm{r} 4$ is characterized by formation of the Mauthner neurons, a pair of large reticulospinal neurons found in fish and amphibians that are involved in the escape response (Fig. 1c). We find that a large percentage of dusp 6 and dusp 2 morphants are missing one or both Mauthner cells (Fig. 1e-j), while a control MO has no effect (Fig. 1d). Furthermore, injecting a combination of both MOs results in an increase in the occurrence of this phenotype (Fig. 1k-m). Additionally, we notice a minor defect in the patterning of the facial motor neurons that normally migrate from $\mathrm{r} 4$ to distinct clusters in the caudal rhombomeres of the hindbrain (Fig. 1n-o). The patterning and clustering of these cells is disrupted in the morphants (Fig. 1p-s). Again, the combination of both MOs results in a more severe phenotype, with some morphants lacking detectable facial motor neurons (Fig. 1t-v). Despite these neuronal defects, the expression of two genes involved in patterning of the early hindbrain appears normal in the morphants (Fig. 1w-y). Additional neurons and markers were examined in the dusp 2 morphants, including the reticulospinal neurons, pERK, pea3, erm, fgf8, valentino, and the abducens motor neurons, with no defects (Additional file 3). These results demonstrate that MOs targeting dusp 6 and dusp 2 disrupt the formation and migration of neurons originating in $\mathrm{r} 4$ of the hindbrain.

\section{Generation of dusp6 and dusp2 germ line mutants}

To investigate the roles of dusp6 and $d u s p 2$ in zebrafish development in greater detail, we set out to generate germ line mutants using the CRISPR/Cas9 genome editing system. We designed two guide RNAs for each gene - one targeted to the $5^{\prime}$ end of the coding sequence and one targeted to the $3^{\prime}$ end (Fig. 2a, Table 1) - with the intention of co-injecting them to delete the sequence between the two target sites. Dusp proteins contain a C-terminal catalytic domain required for substrate recognition and binding [30], as well as an N-terminal rhodanese-homology domain. Although the latter domain is catalytically inactive [31, 32], we nevertheless elected to delete both domains with the goal of generating null alleles. Hence, guide RNA target sequences were chosen based on their proximity to the start and stop codons of the coding sequence, and also by the requirement for a protospacer adjacent motif (PAM) sequence (NGG) at the 3' end of each target site (Fig. 2a, Table 1).

We injected in vitro transcribed guide RNAs and mRNA encoding cas 9 into early 1 -cell stage embryos to test if the guide RNAs were functional. To this end, we prepared genomic DNA from pools of injected embryos at $24 \mathrm{hpf}$ and analyzed the target sites by PCR. Using primers that anneal outside the guide RNA target sites (Primers dusp61/dusp6-2 and dusp2-1/dusp2-3; Fig. 2a, Additional file 2), we detected bands of approximately 400-600 bp (Fig. 2b), indicating the presence of large deletions created by both the $d u s p 6$ and $d u s p 2$ guide RNA pairs. Each guide RNA pair was then injected into several hundred embryos that were raised to adulthood as the F0 generation (Fig. 2c). This F0 generation is mosaic and each individual fish may carry more than one mutant allele for the same gene. We therefore identified founder fish carrying germ line mutations by crossing F0 individuals to wildtype fish and screening for deletions in the resulting offspring (Fig. 2c) using the same PCR primers (Primers dusp6-1/dusp6-2 and dusp2-1/dusp2-3; Fig. 2a, Additional file 2). F0 founders that were positive for germ line mutations were crossed to wildtype fish and the offspring raised to adulthood followed by genotyping to identify heterozygous F1 carriers.

For dusp6, two F0 founders with germ line mutations were identified out of 23 fish tested (Table 1). One founder 

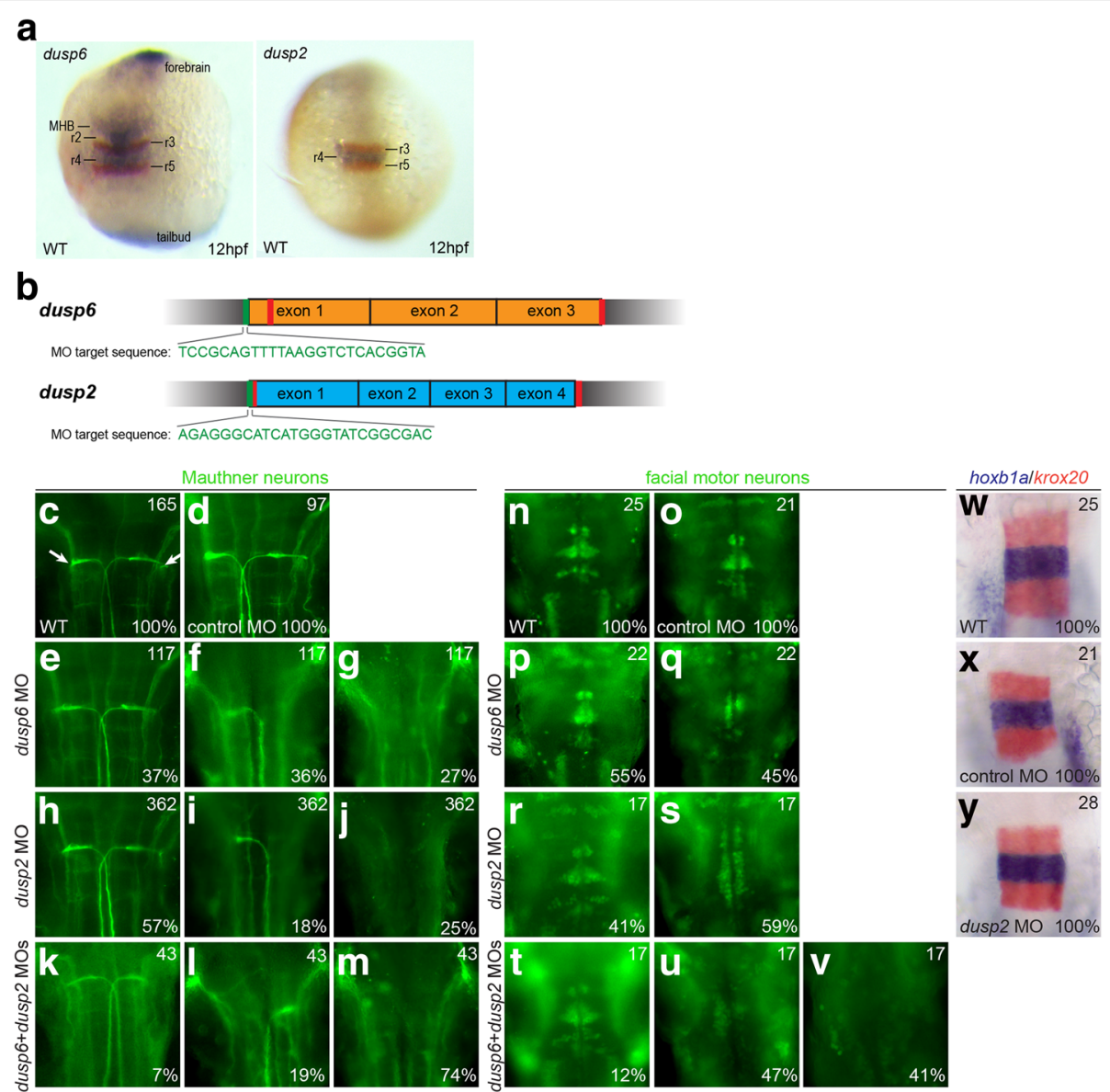

Fig. 1 Knockdown of dusp2 and dusp6 via MO yields a hindbrain phenotype. a $12 \mathrm{hpf}$ wildtype embryos were assayed by in situ hybridization for expression of krox20 (red stain) and dusp6 (blue stain in left panel) or dusp2 (blue stain in right panel). b Schematic of genomic sequence for dusp6 and dusp2. Red vertical lines indicate CRISPR target sites and green vertical lines indicate MO target sites. c-v 48hpf wildtype (c, $\mathbf{n}$ ), control MO-injected (d, o), dusp6 MO-injected (e-g, p-q), dusp2 MO-injected (h-j, r-s), and dusp6 + dusp2 MO-injected (k-m, t-v) embryos were assayed by immunostaining for differentiation of Mauthner neurons (3A10 staining in $\mathbf{c - m}$ ) and facial motor neurons (Islet1/2 staining in $\mathbf{n}-\mathbf{v}$ ). w-y $18 \mathrm{hpf}$ wildtype (w), control MO-injected $(\mathbf{x})$ and dusp2 MO-injected $(\mathbf{y})$ embryos were assayed by in situ hybridization for expression of krox20 (red stain) and hoxbla (blue stain). Numbers in top right corner of each panel indicate the total number of embryos assayed for that condition. Numbers in bottom right corner indicate percent of embryos with the phenotype shown. All embryos are in dorsal view with anterior to the top. Embryos in (a) are whole-mounts, while embryos in (c-y) are flat-mounted and show only the central hindbrain region

$\left(\right.$ dusp $\left.6^{\text {um239 }}\right)$ carried a mutant allele with a $2.2 \mathrm{~kb}$ deletion within the coding sequence of the dusp 6 gene. The exact nucleotides deleted were determined by sequencing both genomic DNA and cDNA (Additional file 4). This large deletion appears to be the product of two double strand breaks as was expected. Translation of this sequence predicts a 63-amino-acid protein with no known protein domains (Fig. 2d). This founder transmitted this mutation to $13 \%$ of its offspring (Table 2). A second founder (dus$p 6^{\text {um286}}$ ) carried a mutant allele with a $1.3 \mathrm{~kb}$ deletion spanning exons 2 and 3 of the dusp 6 gene. We suspect that the $5^{\prime}$ guide RNA did not cause a break in this case, and instead the 3 ' guide RNA generated a cut that was not properly repaired resulting in a smaller deletion. Translation of the resulting sequence predicts a 135amino-acid protein that lacks the catalytic domain (Fig.
$2 \mathrm{~d})$. This founder transmitted this mutation to $24 \%$ of its offspring (Table 2).

For dusp2, three F0 founders with germ line mutations were identified out of 23 fish tested (Table 1). Each of these founders arose from an independent injection, but interestingly, all three carried the same mutant allele containing a $2.8 \mathrm{~kb}$ deletion within the coding sequence. Again, the exact nucleotides deleted were determined by sequencing of genomic DNA (Additional file 4). The mutant allele translates to produce a 57 -amino-acid protein that lacks any known protein domains (Fig. 2d). The first dusp $2^{u m 287}$ founder transmitted this mutation to $18 \%$ of its offspring (Table 2). The two additional founders were positive for a deletion by PCR, but we were unable to identify any heterozygous carriers from their offspring. Hence, we have generated two dusp6 and one 


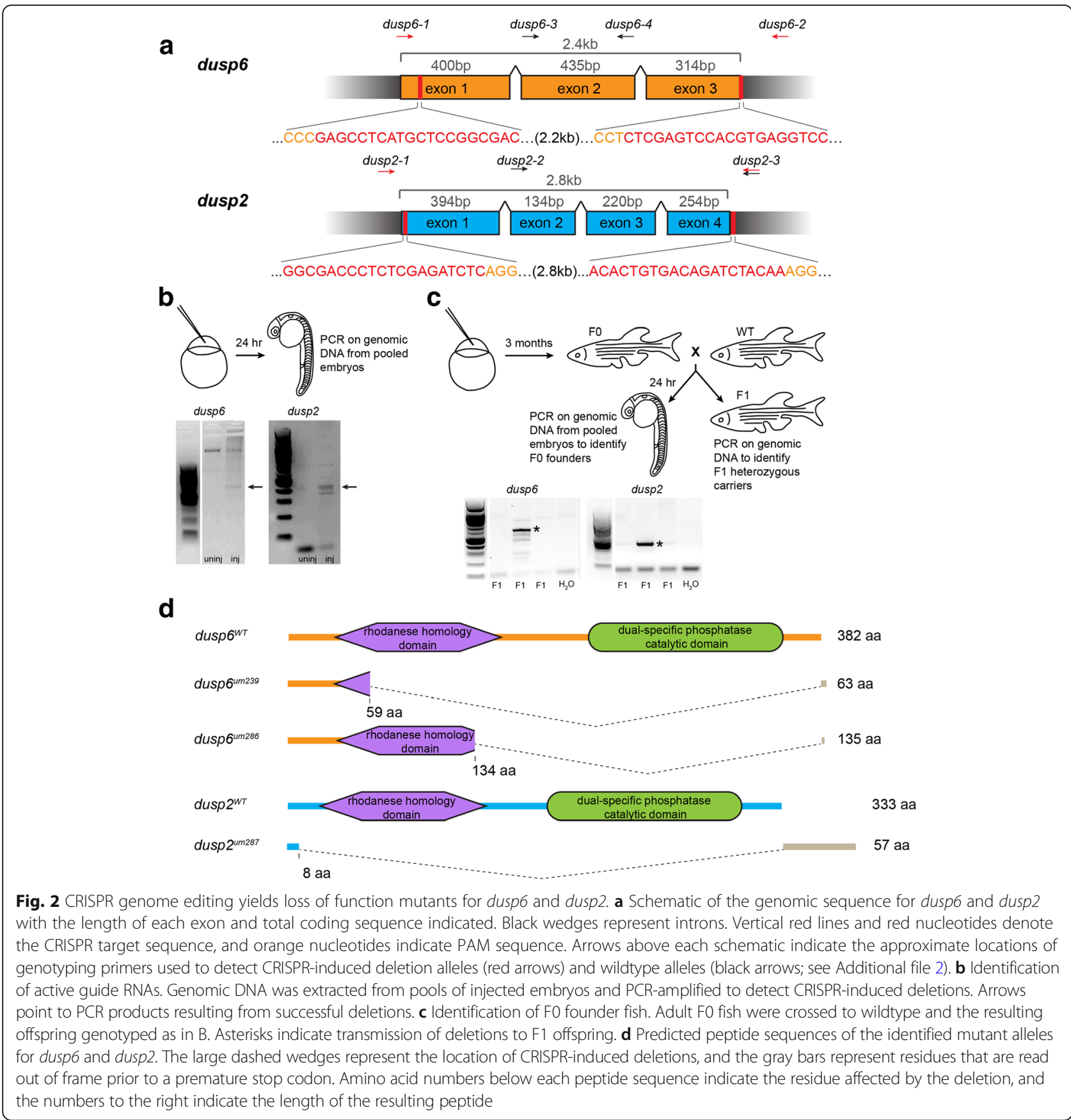

Table 2 Characteristics of dusp6 and dusp2 mutant alleles

\begin{tabular}{|c|c|c|}
\hline allele ID & Transmission frequency $^{a}$ & Size of deletion ${ }^{b}$ \\
\hline dusp6 $6^{\text {um239 }}$ & $13.3 \%$ & $2263 \mathrm{bp}$ \\
\hline dusp $6^{\text {um286 }}$ & $23.8 \%$ & $1308 \mathrm{bp}$ \\
\hline dusp2 $2^{\text {um287 }}$ & $18.2 \%$ & $2855 \mathrm{bp}$ \\
\hline
\end{tabular}

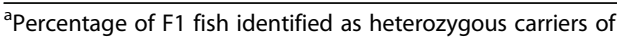
CRISPR-induced deletions

${ }^{\mathrm{b}}$ Total number of nucleotides deleted from the genomic sequence dusp 2 alleles that are predicted to lack phosphatase activity.

\section{dusp6 and dusp2 mutants do not recapitulate the morphant phenotype}

While breeding the mutant lines, we found that both dusp6 and dusp2 homozygous mutants are viable. Accordingly, crosses between double heterozygous dusp $2^{u m 287 /+} ;$ dusp $6^{u m 286 /+}$ carriers produced offspring with all genotypes represented at the expected ratios and all could be raised to adulthood (Fig. 3a). We took 
advantage of the homozygous mutant viability and used embryos derived from crosses between double homozygous mutant parents $\left(\right.$ dusp $2^{\text {um287/um287}} ;$ dusp $6^{\text {um286/um286 }}$ female crossed to dusp $2^{\text {um287/um287}} ;$ dusp $6^{\text {um } 286 / u m 286}$ male) for RNA-seq analysis to identify global changes in gene expression resulting from simultaneous loss of both $d u s p 6$ and dusp2 (Fig. 3b). Since dusp6 and dusp 2 are expressed in multiple tissues at segmentation stages, we extracted RNA from pools of 18hpf whole embryos to generate the RNA-seq libraries.

RNA-seq analysis yielded 673 genes that are differentially-expressed between wildtype and mutant embryos out of 23,150 total genes with mapped reads.
Of those that are differentially-expressed, 334 are upregulated and 339 are down-regulated in the mutants (Fig. 3c). We selected 23 differentially expressed genes for validation by quantitative PCR (qPCR) on independently prepared cDNA samples collected from sibling embryos. We find that the expression changes observed by RNA-seq are confirmed by qPCR analysis for 18 of these genes (78\%; Fig. 3d).

Next, we narrowed the number of candidate genes down to 504 by pursuing only those with a Zebrafish Information Network (ZFIN, [33]) gene ID number, as these have available information regarding their expression pattern. For this list of differentially-expressed genes,

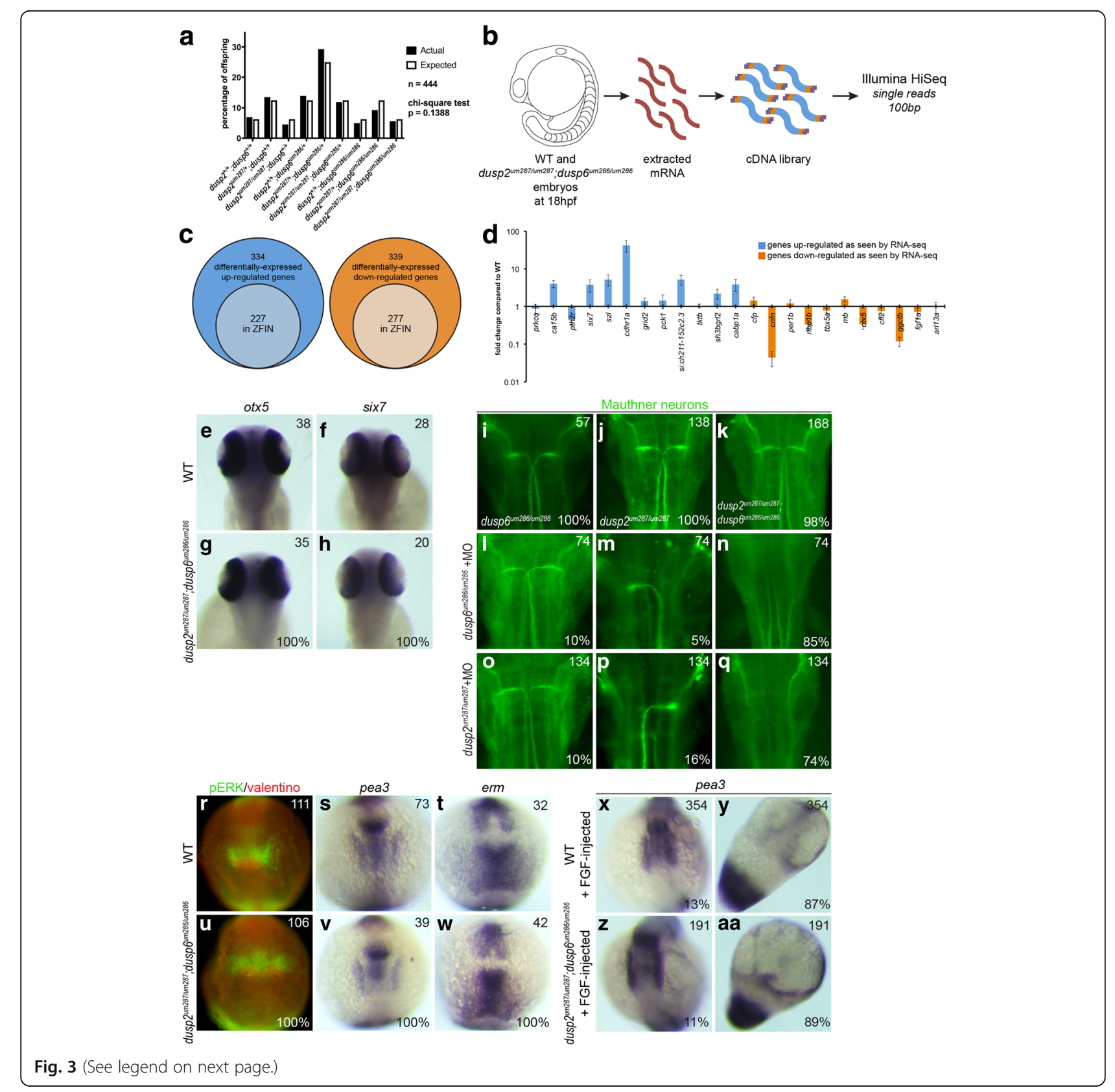


(See figure on previous page.)

Fig. 3 Loss of dusp6 and dusp2 does not impact early development. a Offspring from crosses between double heterozygous dusp $2^{\text {um } 287 /}$ ${ }^{+} ;$dusp $6^{\text {um286/+ }}$ males and females were raised to adulthood and genotyped in order to determine the percentage of each possible genotype in surviving fish. A chi-square test indicates no significant statistical difference between the actual and expected Mendelian ratios. $\mathbf{b}$ Outline of RNAseq library production from wildtype and dusp2/dusp6 double mutant (derived from crosses between dusp $2^{\text {um } 287 / \text { um } 287}$; dusp $6^{\text {um } 286 / u m 286}$ females

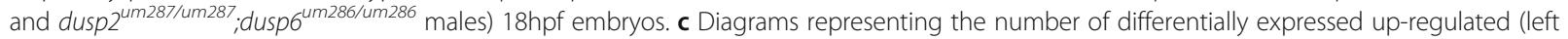
circles) and down-regulated (right circles) genes in dusp2/dusp6 double mutant (derived from crosses between dusp2 $2^{\text {um } 287 / u m 287} ;$ dusp $6^{\text {um2 } 286 / u m 286}$ females and dusp2 $2^{\text {um287/um287; }}$ dusp6 $6^{\text {um286/um286 }}$ males) versus wildtype embryos. Inner circles indicate the subset of genes annotated in ZFIN. d 23 genes identified as differentially expressed by RNA-seq were re-examined by qPCR on cDNA derived from wildtype versus dusp2/dusp6 double

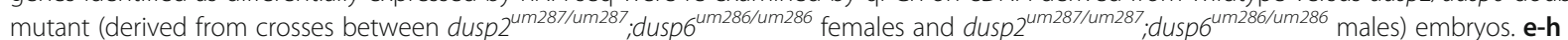

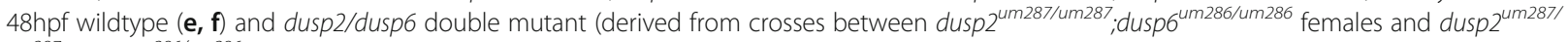
${ }_{\text {um287; }}$ dusp6 $6^{\text {um286/um286 }}$ males; $\left.\mathbf{g}, \mathbf{h}\right)$ embryos were assayed for changes in $\operatorname{otx} 5(\mathbf{e}, \mathbf{g})$ and $\operatorname{six} 7(\mathbf{f}, \mathbf{h})$ expression by in situ hybridization. i-q Uninjected (i-k) or MO-injected (I-q) dusp6 mutant (derived from crosses between dusp6 ${ }^{\text {um286/um286 females and dusp6 }}{ }^{\text {um286/um286 }}$ males; i), dusp2 mutant (derived from crosses between dusp2 ${ }^{\text {um } 287 / u m 287}$ females and dusp $2^{\text {um2 } 287 / u m 287}$ males; j), and dusp2/dusp6 double mutant (derived from crosses between

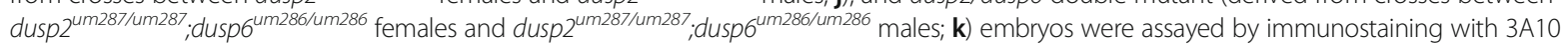
antibody to detect the Mauthner neurons at 48hpf. $\mathbf{r}-\mathbf{w} 12 \mathrm{hpf}$ wildtype $(\mathbf{r}, \mathbf{s}, \mathbf{t})$ and dusp2/dusp6 double mutant (derived from crosses between

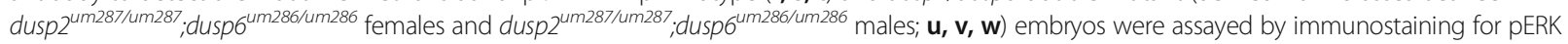
(green in $\mathbf{r}$, $\mathbf{u}$; red counterstain detects the Valentino transcription factor), as well as by in situ hybridization for expression of pea3 ( $\mathbf{s}, \mathbf{v})$, and erm (t, $\mathbf{w})$.

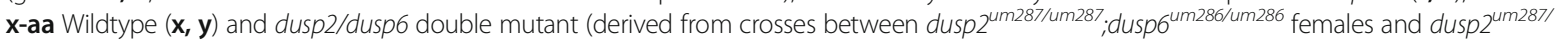

um287; dusp $6^{\text {um286/um286 }}$ males; $\mathbf{z}$, aa) embryos were injected with fgf8 mRNA and the expression pattern of pea3 visualized by in situ hybridization. All embryos are in dorsal view with anterior to the top. Numbers in top right corner of each panel indicate the total number of embryos assayed for that condition. Numbers in bottom right corner indicate percent of embryos with the phenotype shown

we examined whether there is an enrichment in genes that function within particular pathways, specifically the ERK signaling pathway or another MAPK pathway. Although the PANTHER gene ontology classification system [34-36] grouped 124 of the up- and down-regulated genes into 44 different pathways, there is no clear enrichment for any singular pathway (Additional file 5). We next reasoned that genes expressed in the same regions as dusp6 and/or dusp 2 would be the best candidates for genes affected in the mutant lines. Using ZFIN's gene expression database for wildtype fish [37], we analyzed the body structures in which the candidate genes are expressed, with a focus on the regions containing dusp 6 and dusp2. Of the 504 genes, 97 are expressed in 25 different structures that overlap with dusp6 and dusp 2 expression (Additional file 6). We selected two genes (otx5 and six7) that are expressed in the same regions of the forebrain as dusp 6 and dusp 2 and that were also validated by $\mathrm{qPCR}$, but we were unable to detect any change in expression of these genes using in situ hybridization (Fig. 3e-h).

The lack of an apparent phenotype in dusp germ line mutants led us to examine if these mutants recapitulate the loss of Mauthner cells observed in dusp morphants. Strikingly, Fig. 3i-k shows that Mauthner neurons form normally in dusp6 mutant embryos (derived from crosses between dusp $6^{\text {um286/um286 }}$ females and dusp $6^{\text {um286/um } 286}$ males) and in dusp 2 mutant embryos (derived from crosses between dusp $2^{\text {um } 287 / u m 287}$ females and

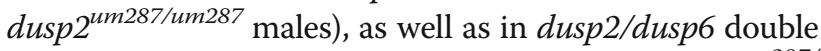
mutants (derived from crosses between dusp $2^{u m 287 /}$ ${ }^{\text {um } 287}$; dusp $6^{\text {um } 286 / \text { um } 286}$ females and dusp $2^{\text {um } 287 /}$ ${ }^{\text {um } 287}$; dusp $6^{\text {um } 286 / \text { um } 286}$ males). To examine the cause of this discrepancy further, we injected MOs into the respective mutant line and find that Mauthner cells are lost (Fig. 3l-q). Because the mutant embryos lack the sequences encoding each phosphatase, the loss of Mauthner cells cannot be due to the MOs affecting dusp gene expression, but is likely caused by an offtarget effect. Since a previous study reported defective bmp4 and chordin expression in dusp6 morphants [20], we also examined a variety of other genes involved in early embryonic patterning, including krox $20, f g f 3, f g f 8, b m p 2 b$, bmp4, chordin, and noggin1, but detect no changes in expression in dusp2/dusp6 double mutants (derived from

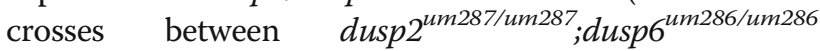
females and dusp $2^{\text {um } 287 / u m 287} ;$ dusp $6^{\text {um } 286 / \text { um } 286}$ males; Additional file 7). We conclude that zebrafish dorsoventral patterning and Mauthner cell formation is independent of dusp 6 and dusp 2 activity.

To further address the lack of a phenotype, we next investigated the level of pERK (the primary substrate for both Dusp6 and Dusp2) during early segmentation, when both phosphatases are expressed. dusp $2 / d u s p 6$ double mutant embryos (derived from crosses between dusp $2^{\text {um287/um287}} ;$ dusp $6^{\text {um286/um286 females and }}$ dusp $2^{\text {um287/um } 287} ;$ dusp $6^{\text {um 286/um286 }}$ males) stained with an anti-pERK antibody, and counterstained with an antiValentino antibody marking $\mathrm{r} 5$ and $\mathrm{r} 6$ of the hindbrain, show no differences in intensity or location of pERK within the hindbrain or other regions of the embryo compared to wildtype embryos (Fig. 3r, u). We also examined the expression patterns of two ERK target genes, pea3 and erm, of which neither is affected in the mutants (Fig. 3s-t, v-w). Since key signaling pathways, such as the ERK signaling pathway, are held under many levels of regulation [7, 38-42], we considered the possibility that other forms of control could be compensating for the loss of dusp 6 and dusp2. Accordingly, we 
hypothesized that challenging the pathway by exposure to higher levels of ligand might expose a defect in the mutants. To test this, we injected wildtype and dusp2/ dusp6 double mutant (derived from crosses between dusp $2^{\text {um287/um287 }}$;dusp $6^{\text {um286/um286 females and }}$ dusp $2^{\text {um287/um287}} ;$ dusp $6^{\text {um286/um286 }}$ males) embryos with fgfo mRNA, raised them to the early segmentation stages, and then examined the expression pattern of the ERK target gene pea3. While excess $f g f 8$ proved to have a gross effect on early embryonic development and morphology, we did not observe a difference in the effect between wildtype and mutant embryos (Fig. 3x-aa). Hence, despite validated gene expression changes in the mutants, dusp6 and dusp 2 mutants do not recapitulate the morphant phenotype.

\section{Homozygous dusp6 mutant embryos have reduced viability through gastrulation}

During our analysis, we noticed that the offspring of dusp6 homozygous mutant parents has reduced viability during the first $10 \mathrm{~h}$ after fertilization. To examine this effect further, wildtype and dusp 6 mutant (derived from crosses between dusp $6^{\text {um239/um239 }}$ females and dusp $6^{\text {um } 239 / u m 239}$ males) clutches were collected and the number of live embryos counted at $1 \mathrm{hpf}$ and $10 \mathrm{hpf}$. We routinely observe that a small percentage (approximately 5\%) of wildtype embryos die by the end of gastrulation, but the homozygous dusp6 mutant embryos show a significant decrease in viability with only approximately $50 \%$ of embryos surviving to $10 \mathrm{hpf}$ (Fig. 4a). We also examined the viability of dusp2/ dusp6 double mutant embryos (derived from crosses between $d u s p 2^{\text {um287/um287}} ;$ dusp $6^{\text {um286/um286 }}$ mutant females

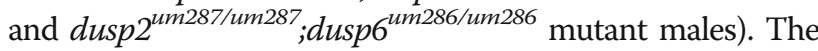
dusp 2/dusp6 double mutant clutches show a decrease in viability (Fig. 4b) that is indistinguishable from the dusp $6^{\text {um239/um } 239}$ mutant clutches (Additional file 8A), indicating that loss of $d u s p 2$ does not decrease viability further and demonstrating that the dusp $6^{u m 239}$ and $d u s p 6^{u m 286}$ alleles produce quantitatively similar phenotypes. Lastly, we examined if having one mutant parent is sufficient for reduced viability. To address this, we crossed a wildtype female to a dusp $2^{u m 287 / u m 287} ; d u s p 6^{u m 286 / u m 286}$ mutant male or a wildtype male to a dusp $2^{\text {um287/um287} ; d u s p 6^{\text {um286/um286 }}}$ mutant female. The survival of embryos from these crosses, while somewhat variable from clutch to clutch, is not statistically different than that of wildtype embryos (Fig. 4c; Additional file $8 \mathrm{~A}$ ), indicating that reduced viability is apparent only when both parents are mutant.

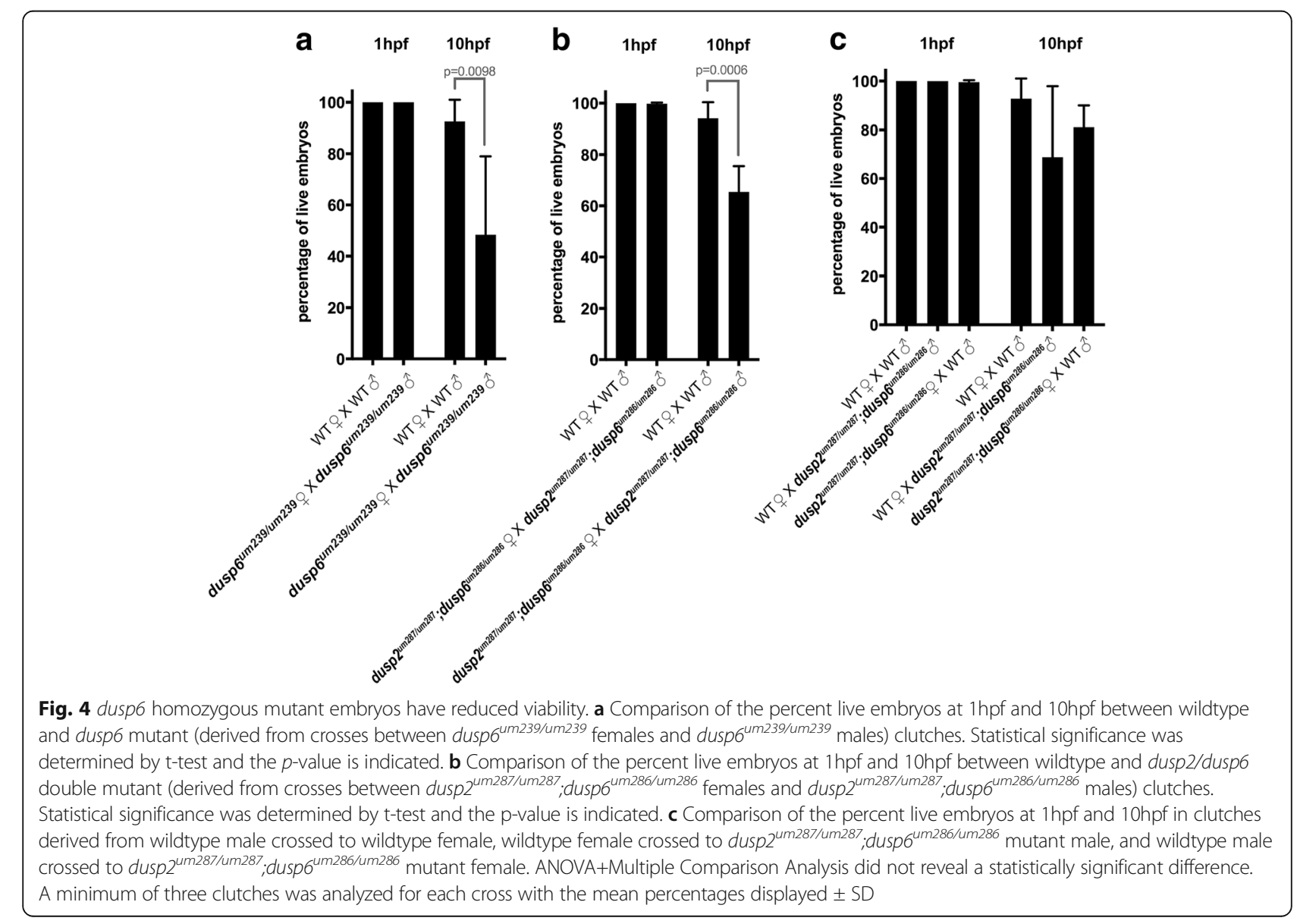




\section{A fraction of homozygous dusp6 mutant embryos stall at the first cell division}

To further characterize the reduced viability of dusp6 mutants, we collected clutches of wildtype and homozygous dusp6 mutant (derived from crosses between dusp $6^{\text {um239/um239 }}$ females and dusp $6^{\text {um239/um239 }}$ males) embryos and monitored them throughout the cleavage, blastula, and gastrula stages (Fig. 5a). As expected, we again found that $50 \%$ of dusp 6 mutant embryos die by 10hpf. Notably, in contrast to wildtype embryos that had all undergone at least one round of cell division by $1 \mathrm{hpf}$, approximately $40-50 \%$ of the dusp 6 mutant embryos remained at the 1 -cell stage at $1 \mathrm{hpf}$. We refer to these as 'stalled' embryos and we monitored their development for the subsequent stages. We find that all of the stalled embryos remain at the 1-cell stage over the next 8 to $10 \mathrm{~h}$ until they eventually die. We noticed that some of the stalled embryos proceed to develop a slight cleavage furrow, but they appear unable to complete the process of cell division, and will later return to the smooth cell surface typically seen at the 1-cell stage. When monitoring dusp 2/dusp6 double mutant clutches (derived from crosses between dusp $2^{\text {um287/um287} ; d u s p 6^{\text {um 286/um286 }}}$ females and dusp $2^{\text {um287/um287}} ;$ dusp $6^{\text {um2286/um286 }}$ males), we find that they show an identical phenotype to dusp $6^{\text {um239/um239 }}$ mutants (Fig. 5a). This is true both in terms of the detailed phenotype (stalling at the 1-cell stage with occasional incipient cleavage furrows), its onset (starting at the 1-cell stage) and duration (all stalled embryos have died by $10 \mathrm{hpf}$ ), demonstrating that the $d u s p 6^{u m 239}$ and $d u s p 6^{u m 286}$ alleles produce qualitatively indistinguishable phenotypes. We also examined clutches from a wildtype female crossed to a $d u s p 2^{u m 287 /}$ ${ }^{\text {um } 287}$; dusp $6^{\text {um286/um286 }}$ mutant male and a wildtype male

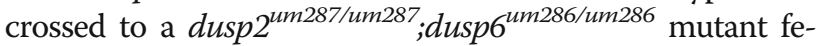
male. These exhibited less severe effects (Additional file $8 \mathrm{~B}$ ), further supporting our conclusion that both parents need to be mutant to significantly affect embryo viability.

Although the stalled embryos appear unable to complete cell division, it is unclear if they are progressing through the cell cycle. To address this, we visualized the nuclei of wildtype and dusp2/dusp6 double mutant (derived from

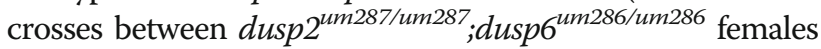
and dusp $2^{\text {um287/um287}} ;$ dusp $6^{\text {um286/um286 males) embryos }}$ using DAPI at 1hpf. At this time point, wildtype and healthy dusp $2 / d u s p 6$ double mutant embryos are entering the 8-cell stage, while stalled dusp $2 / d u s p 6$ double mutant embryos remain at the 1-cell stage. Accordingly, wildtype and healthy dusp $2 / d u s p 6$ double mutant embryos contain eight DAPI-positive nuclei with varying degrees of condensation likely depending on their position in the cell cycle at the time of fixation (Fig. 5b, c). In contrast, the stalled embryos contain a single large and disorganized DAPIpositive nucleus (Fig. 5d). We conclude that stalled dusp2/ dusp6 double mutant embryos are unable to complete the cell cycle.

To test at what point of the cell cycle the stalled embryos are arresting, we used a phospho-histone H3 antibody to detect mitotic nuclei. Histone $\mathrm{H} 3$ becomes phosphorylated at serine 11 during the end of the G2 phase and the early stages of mitosis [43]. Because the first several cell cycles in the developing zebrafish embryo lack G1 and G2 phases [44], positive staining with this antibody should indicate nuclei that are in mitosis and not in interphase. Since the embryos in this experiment derived from natural matings, we expected to see some embryos in mitosis and some in interphase. At 1hpf, when normally developing embryos should enter the 8 -cell stage, we find that $44 \%$ of wildtype embryos and $31 \%$ of healthy dusp $2 /$ dusp 6 double mutant embryos (derived from crosses between dusp2 $2^{u m 287 /}$

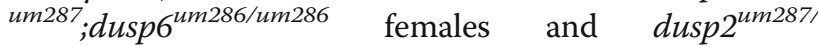
${ }^{u m} 287$; dusp $6^{\text {um } 286 / u m 286}$ males) are mitotic, while the remaining embryos are in interphase (Fig. 5e-h-i). In contrast, at $1 \mathrm{hpf}$ all stalled dusp $2 /$ dusp 6 double mutant embryos contained a single nucleus that is positively stained with the phospho-histone H3 antibody (Fig. 5g). Since the first round of mitosis should have begun at approximately $30 \mathrm{~min}$ post fertilization, these embryos must have been stalled in mitosis for at least $30 \mathrm{~min}$ prior to fixation. Additionally, all of the stalled embryos contained only one nucleus, as seen by the DAPI and phospho-histone H3 staining, indicating that they do not proceed to anaphase when the sister chromatids are pulled apart. Indeed, separated chromatids are commonly seen in wildtype and healthy dusp $2 / d u s p 6$ double mutant embryos at $1 \mathrm{hpf}$ (Additional file 9), but are never observed in stalled embryos.

We next examined whether the stalled embryos are fertilized. In the few minutes following fertilization, the maternal and paternal pronuclei condense, migrate towards each other, and merge, allowing the zygote to enter the cell cycle. Hence, the presence of two pronuclei indicates that an embryo has been fertilized. To visualize fertilization, we fixed wildtype and dusp2/ dusp6 double mutant (derived from crosses between dusp $2^{\text {um287/um287}}$;dusp $6^{\text {um286/um286 females and }}$ dusp $2^{\text {um287/um287}} ;$ dusp $6^{\text {um286/um286 males) embryos }}$ 10 min post fertilization and stained them with DAPI. Because pronuclear fusion is very rapid and the embryos are collected from natural matings, it is difficult to catch all pronuclei prior to fusion. Accordingly, we find $79 \%$ of wildtype embryos contain two detectable pronuclei at $10 \mathrm{~min}$ post fertilization, indicating that these embryos are fertilized (Fig. $5 \mathbf{j}-\mathrm{n}$ ). At this early time point, we cannot distinguish between $d u s p 2 / d u s p 6$ double mutant embryos that are healthy and those that will stall at the 1 -cell stage. However, if the stalled embryos were not 

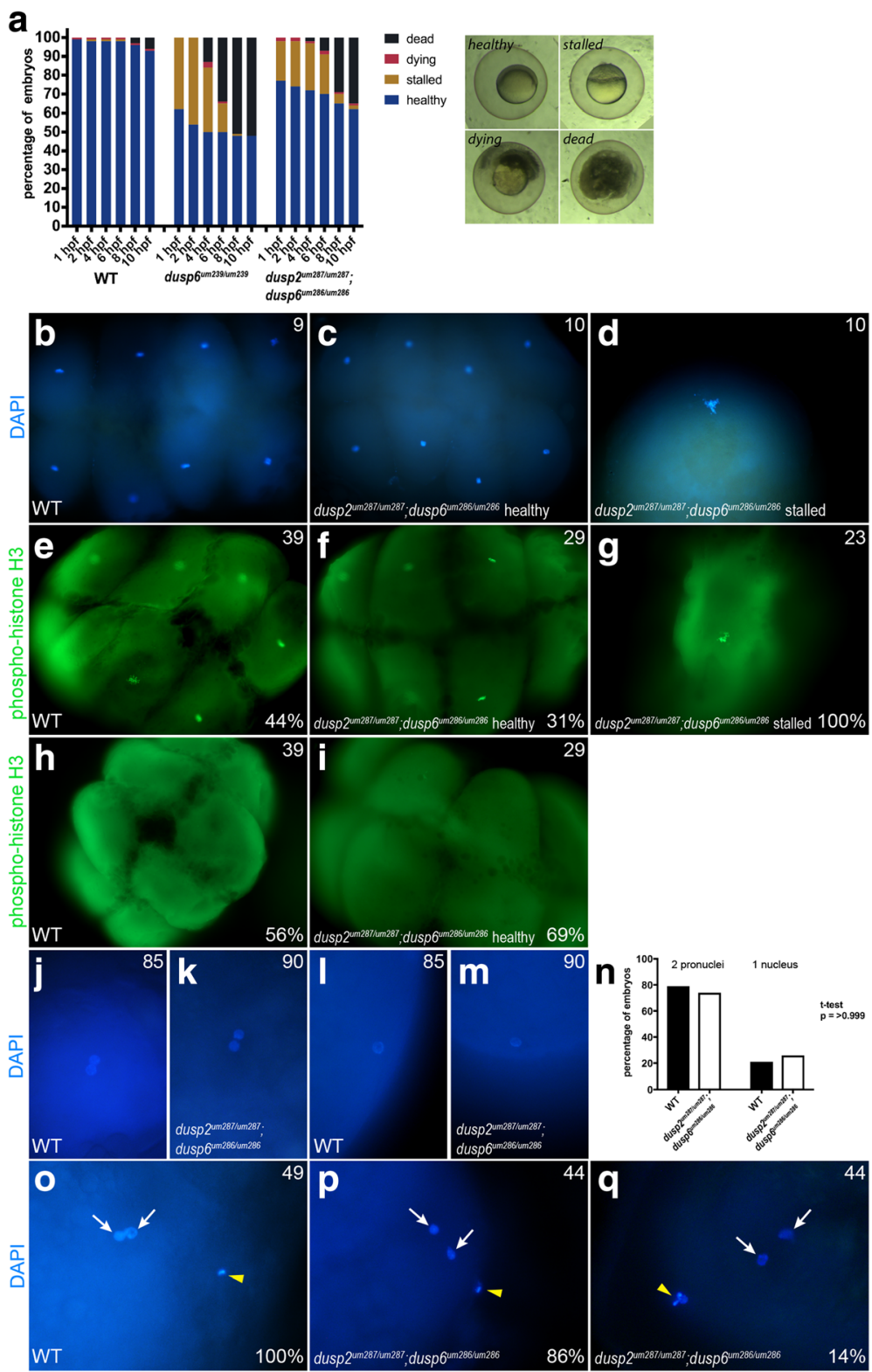

Fig. 5 A fraction of dusp6 homozygous mutant embryos stall at the first cell division. a Wildtype, dusp6 mutant (derived from crosses between dusp $6^{\text {um239/um } 239}$ females and dusp $6^{\text {um239/um239 }}$ males), and dusp2/dusp6 double mutant (derived from crosses between dusp2 ${ }^{\text {um } 287 /}$

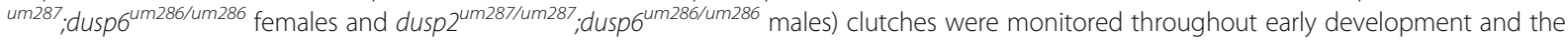
health of each embryo scored at each time point according to the brightfield images to the right (a minimum of three clutches were scored for each cross). Average cumulative counts are shown. b-d DAPI staining on 1 hpf wildtype and dusp2/dusp6 double mutant (derived from crosses

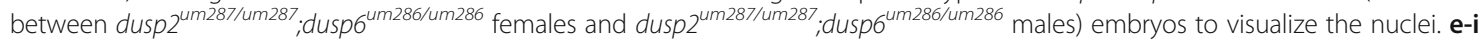

Immunostaining for phospho-histone $\mathrm{H} 3$ to visualize mitotic nuclei of wildtype and dusp2/dusp6 double mutant (derived from crosses between

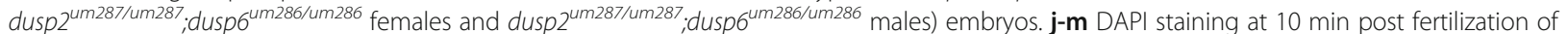

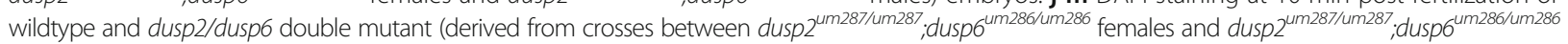
males) embryos to visualize the early pronuclei. $\mathbf{n}$ Quantification of wildtype and dusp2/dusp6 double mutant (derived from crosses between dusp2 ${ }^{\text {um287/ }}$

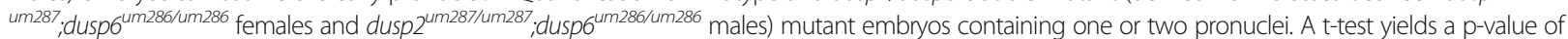
> 0.999 indicating no significant statistical difference. o-q DAPI staining to detect polar bodies in wildtype and dusp2/dusp6 double mutant (derived from

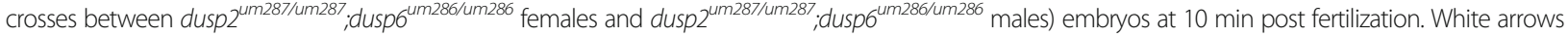
indicate pronuclei and yellow arrowheads indicate polar bodies in (o-q). Numbers in top right corner of each panel indicate the total number of embryos assayed for that condition. Numbers in bottom right corner indicate percent of embryos with the phenotype shown 
fertilized, we would expect to see an approximate 50\% reduction in dusp2/dusp6 double mutant embryos with two pronuclei, since we know that $50 \%$ of them will stall (Fig. 5a). Instead, we find that $74 \%$ of dusp $2 / d u s p 6$ double mutant embryos contain two detectable pronuclei (Fig. $5 k-n$ ), indicating that these embryos are fertilized at the same rate as wildtype embryos. A t-test confirms that there is no significant difference in the fraction of embryos with two pronuclei from wildtype and dusp $2 /$ dusp 6 double mutant clutches (Fig. 5n).

DAPI staining at $10 \mathrm{~min}$ post fertilization also labels the polar bodies and we noticed that some dusp2/dusp6 double mutant (derived from crosses between dusp $2^{\text {um287/um287 }}$; dusp $6^{\text {um286/um } 286}$ females and dusp $2^{\text {um287/um } 287} ;$ dusp $6^{\text {um 286/um } 286}$ males) embryos have large and disorganized polar bodies (Fig. 5o-q). The frequency of abnormal polar bodies (14\%) is lower than the frequency of stalled embryos (approximately 50\%) and the polar bodies in dusp2/dusp 6 double mutants do not persist longer than in wildtype embryos (both are degraded by $1 \mathrm{hpf}$ ), likely ruling out a role for abnormal polar bodies in the stalling of mutant embryos.

We conclude that dusp $2 / d u s p 6$ double mutant embryos are fertilized, but approximately $50 \%$ of them stall during mitosis of the first embryonic cell division. These embryos remain arrested in the early stages of mitosis for several hours until they die prior to the end of gastrulation.

\section{dusp6 is expressed in zebrafish ovaries and testes}

Our analysis revealed that approximately half of dusp2/ dusp6 double mutant embryos stall during the first embryonic cell division. The first several zygotic cell cycles precede activation of the zygotic genome, which occurs at $3-4 \mathrm{hpf}$ in zebrafish embryos, and is therefore largely controlled by maternally deposited components supplied during oocyte maturation in the ovary. We therefore determined if $d u s p 6$ transcripts are detectable in the ovary and in the early fertilized embryo. We find that dusp6 is robustly expressed in the ovary, albeit at somewhat lower levels than in other adult tissues (Fig. 6a). In contrast, dusp6 is detected at very low levels at maternally controlled stages of embryogenesis (2.5hpf) relative to zygotically controlled stages (6hpf; Fig. 6b), in agreement with a previous report that dusp6 and dusp 2 transcripts are not maternally deposited in zebrafish [45]. In zebrafish, the large oocytes contribute the majority of cellular volume of the ovary while smaller granulosa cells surround the maturing oocytes and provide growth signals, maternal transcripts, and nutrients via gap junctions. Since dusp6 is present at very low levels in fertilized oocytes (Fig. 6b), it is likely that dusp6 is primarily expressed in the granulosa cells, although we cannot exclude the possibility that maternal dusp6 is specifically degraded in oocytes. Interestingly,

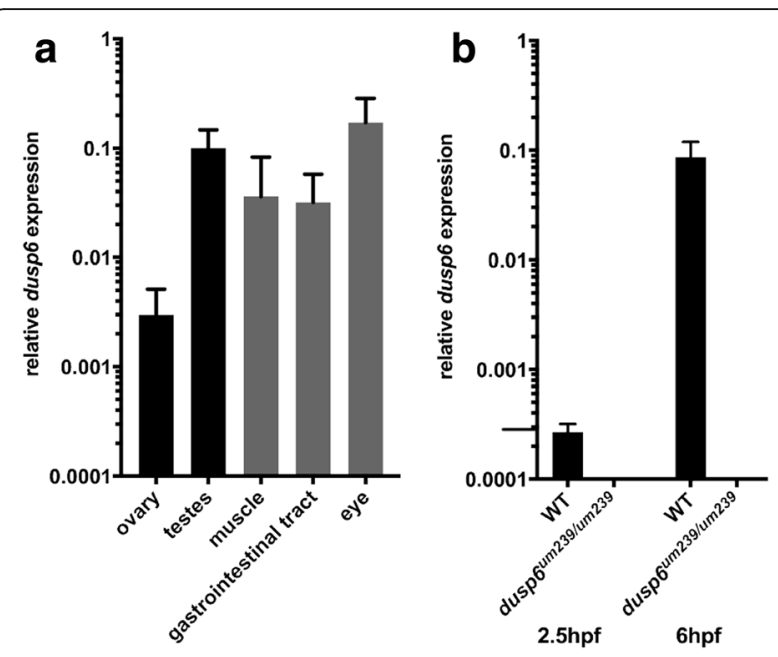

Fig. 6 dusp6 is expressed in ovaries and testes. a dusp6 expression in wildtype adult zebrafish organs was assessed by quantitative RT-PCR using primers dusp6-3/dusp6-4. b dusp6 expression in wildtype and dusp6 mutant (derived from crosses between dusp $6^{\text {um239/um239 females }}$

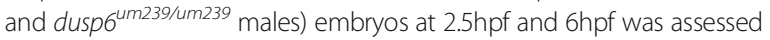
by quantitative RT-PCR using primers dusp6-3/dusp6-4. Results of three independent experiments were normalized to those of $b$-actin and displayed as the mean \pm SD

dusp6 is also expressed in the adult testes (Fig. 6a), consistent with our finding that decreased viability is only detected when both parents are homozygous mutants. Based on the current literature [46], it is likely that dusp6 is expressed in the seminiferous tubules of the testes.

\section{Discussion}

In order to identify a role for $d u s p 6$ and $d u s p 2$ in the developing zebrafish, we generated mutant lines carrying loss of function alleles for these two phosphatases. Our experiments show that $\sim 50 \%$ of off-spring from homozygous dusp6 mutant parents stall at the 1-cell stage and die within the first $10 \mathrm{~h}$ after fertilization. The affected embryos appear to have been fertilized, but are unable to progress through the first cell cycle. The early onset of the phenotype, coupled with the expression of dusp6 in both testes and ovaries, lead us to propose a parental role for dusp6 in permitting zebrafish development to progress past the 1-cell stage. In contrast, loss of $d u s p 2$ function does not affect embryo viability and we have been unable to identify a role for $d u s p 2$ in zebrafish embryogenesis.

\section{dusp6 may act to maintain ERK signaling within a permissive range}

A key observation regarding the stalling of homozygous dusp6 mutant embryos is that only a portion of each clutch is affected (approximately 50\%, Fig. 4a, b, Fig. 5a), demonstrating that the phenotype is incompletely penetrant. This 
implies that not all oocytes and/or sperm produced by homozygous mutant adults are defective, but the basis of the incomplete penetrance is unclear. Differences in expression and concentration of signaling components is known to contribute to variability in signaling intensity among individual cells $[47,48]$, and studies of various signaling pathways have identified roles for redundant regulators in reducing signal noise $[49,50]$. Specifically, there is evidence of cell-to-cell variability in levels of protein kinase signaling, and negative feedback regulators such as Dusp proteins are thought to act to minimize such variations [51]. We therefore hypothesize that dusp6 is required to minimize variations in ERK signaling during gametogenesis and that when dusp6 is lost, a fraction of oocytes and spermatocytes become exposed to ERK signaling outside the permissive range. Under this model, many oocytes and spermatocytes in the mutants would still be exposed to levels of ERK signaling that fall within the permissive range, but a percentage would receive excessive ERK signals leading to abnormal gametogenesis. In support of such a model, while the phenotype of Dusp6 mutant mice is distinct from that in zebrafish (see below), it is nevertheless incompletely penetrant [21], consistent with a more general role for dusp6 in maintaining a permissive range of ERK activity.

However, the possibility remains that the incomplete penetrance results from other causes, such as variations in genetic background, epigenetic factors, individual variations in expressivity, or partial compensation from other regulators. In particular, multiple studies have noted that dusp6 is expressed in many of the same regions of the zebrafish as FGF ligands [7, 10, 41, 52, 53]. Several other proteins known to regulate FGF signaling are also expressed in these regions, and for this reason they have been referred to as the FGF-synexpression group. This group includes other Dusp proteins and phosphatases, members of the Spry family, Sef, and Flrt $[7,41,52,53]$. Since these proteins are present in the same regions and modulate the same pathway, it is possible that they compensate for each other when necessary. To address possible compensation, we analyzed the list of differentially-expressed genes from our RNA-seq experiment to see if other negative ERK modulators of the FGF-synexpression group were up-regulated in dusp 6 and dusp 2 mutants. We did not detect significant changes in expression level of any of these genes, but it remains possible that factors regulated by posttranslational modifications could compensate for the loss of Dusp function.

\section{Parental dusp6 activity is required for zebrafish development to progress past the 1-cell stage}

We note that the mutant phenotype is observed very early - shortly after fertilization - and only in off-spring derived from two homozygous mutant parents. This indicates that the phenotype is independent of the zygotic genome and suggests that $d u s p 6$ activity may be required either in the fertilized zygote (following parental deposition into the gametes), or during gametogenesis in the gonads. We tend not to favor the first possibility since we detect only very low levels of dusp6 in oocytes and sperm are thought not to contain much mRNA, but we cannot exclude the possibility that Dusp6 protein is deposited in oocytes and sperm. In support of the latter model, we find that zebrafish dusp6 is expressed in both ovaries and testes and work in other systems has shown that ERK signaling is essential for gametogenesis. In particular, luteinizing hormone (LH) and follicle stimulating hormone (FSH) are the primary drivers of ovarian follicle growth and stimulators of the granulosa cells surrounding the developing oocyte [54]. A study performed on rat granulosa cells demonstrated that Dusp6 in the granulosa cells keeps ERK inactivated in the absence of FSH [55]. Once maturation is initiated by FSH, PKA is activated through cAMP to inhibit Dusp6, thereby allowing active pERK to accumulate and drive downstream genes promoting oocyte maturation and progression through the meiotic cell cycle [56]. Other genes activated by ERK, such as has 2 and ptgs 2 , are required for the expansion and growth of the granulosa cells within the ovarian follicle [57, 58]. Similarly, spermatogenesis also requires well-coordinated ERK signaling. Cell cycle regulators and condensation factors downstream of ERK are required for proper chromatid separation and condensation maintenance between rounds of meiosis [59-61]. Similar to the granulosa cells of the ovary, Sertoli cells coordinate meiotic progression of the developing spermatocytes and their growth within the testes [62]. Genes downstream of ERK also ensure the integrity of vital tight junctions between the Sertoli cells and spermatocytes during maturation [63]. Hence, zebrafish dusp6 mutants may suffer from excess ERK signaling in these pathways that could in turn affect the activity of downstream targets such as cell cycle regulators. Accordingly, there is evidence that mis-regulation of ERK signaling within the mammalian reproductive system results in abnormal pubertal development and infertility. Similarly, female mice carrying a mutant allele for constitutively active RAS have defects in ovulation, and ERK1/2 mutant female mice are completely infertile [64, 65]. Additionally, congenital hypogonadotropic hypogonadism in humans affects both males and females and has been linked to missense mutations in DUSP6 and other ERK regulators [66].

We note that even if the primary defect in dusp6 mutants occurs during gametogenesis, it does not become manifest until after fertilization. Furthermore, since the phenotype is most pronounced when both parents are mutant, it suggests that an ERK-dependent event requiring both maternal and paternal input is likely affected in the mutant embryos. A plausible candidate for such a process is the formation of 
embryonic centrosomes, which requires centrioles provided by the sperm, but also centrosomal components stored in the oocyte [67]. In this scenario, off-spring from two mutant parents would suffer defects in both maternal and paternal centrosomal components and have more severe phenotypes than off-spring derived from only one mutant parent. Indeed, previous work indicates that ERK signaling is required for centrosome duplication [68, 69] and function [70], at least in cell culture models. Hence, dusp6 activity may be required to keep ERK signaling in a permissive range during gametogenesis and failure to do so may produce defective gametes unable to support the first cell division, possibly due to abnormal centrosome function.

\section{dusp6 mutants reveal species-specific defects}

Previous work revealed that Dusp6 mutant mice exhibit increased pERK and Erm expression, skeletal dwarfism, craniosynostosis, and hearing loss [21]. All of these defects share characteristics with FGF receptor activating mutations, consistent with a role for Dusp6 as a negative regulator of ERK signaling. Similar to dusp6 mutant zebrafish, Dusp6 mutant mice have increased postnatal lethality, with a significant decrease in homozygous mutant pups surviving to weaning age. However, the mouse mutants die at later stages than the zebrafish mutants, suggesting that these phenotypes are somewhat distinct. Also, while previous published analyses of dusp6 morphants demonstrated dorsalization and expansion of neural domains [20] and we initially observed defects in dusp6 morphants, none of these phenotypes are recapitulated in dusp6 germ line mutants. Several recent publications have found similar instances where germ line mutants do not have the same phenotype as the corresponding morphant [23, 24] and, whereas there may be several causes for such discrepancies, our finding that dusp6 MOs produce a phenotype in dusp6 mutants suggests that in our case the morphant phenotype is due to a morpholino off-target effect. Although these analyses suggest that dusp 6 may have species-specific roles, these roles nevertheless appear closely related to ERK signaling in each case. Indeed, it is possible that the observed species differences are due to variable compensation by other ERK-signaling components in different species.

\section{Conclusions}

Our results presented here suggest a parental role for dusp6 in controlling progression through the first cell division in zebrafish. Tight regulation of ERK signaling is vital for these processes and a loss of function dusp 6 allele may result in a shift of active ERK levels. While some gametes develop under permissive conditions in the mutants, others may be exposed to elevated ERK levels and this may negatively impact their maturation. The embryos resulting from the union of a defective egg and defective sperm stall at the 1-cell stage, unable to complete the first mitosis, and die by $10 \mathrm{hpf}$. However, homozygous mutant embryos from unaffected gametes develop with no overt phenotypes, suggesting that other ERK regulators are able to compensate during embryonic development.

\section{Additional files}

Additional file 1: Sequences of oligos to generate CRISPR guide RNAs. The sequence of the genomic target, the PAM sequence, the sequences of the oligos used as a template are shown for each guide RNA. (DOCX $11 \mathrm{~kb}$ )

Additional file 2: Primer sequences to genotype mutants. For both dusp6 and dusp2, two sets of PCR primers were used to genotype: one set to amplify the deletion allele and one set to amplify the wildtype allele. (DOCX $13 \mathrm{~kb}$ )

Additional file 3: Additional neuronal and patterning markers examined in dusp2 morphants. Wildtype, control MO-injected, and dusp2 $\mathrm{MO}$-injected embryos were analyzed by in situ hybridization for the expression of pea3, erm, fgf8, and valentino and by immunostaining to visualize the reticulospinal neurons, pERK, and the abducens motor neurons. (TIFF $3043 \mathrm{~kb}$ )

Additional file 4: Nucleotide and predicted amino acid sequence of mutant alleles. A, B. Nucleotide and predicted amino acid sequence of dusp6 (A) and dusp2 (B) mutant alleles. Genomic DNA was amplified using primer pairs dusp6-1/dusp6-2 and dusp2-1/dusp2-3 (See Fig. 2a and Additional file 2). Uppercase nucleotides indicate the coding sequence and lowercase nucleotides represent the UTR. Green and red nucleotides indicate translation start and stop sites, respectively. Ellipses (...) indicate the continuation of wild type sequences. Dashes (-) indicate deletions and blue nucleotides indicate insertions in the mutant alleles. Predicted protein sequences are indicated in one letter IUPAC code with purple highlighting the rhodanese homology domain and green highlighting the catalytic domain. Asterisks denote the stop codon. Orange residues are predicted to be encoded by the mutant alleles before encountering a stop codon. $C$. Transcripts are produced in dusp6 mutant embryos. CDNA was amplified from homozygous mutant dusp6 embryos by end-point PCR using the same primers as in A. Sequencing of these transcripts identified the same lesions detected in genomic DNA in A. Dashed lines indicate where gel was cut. (TIFF $1730 \mathrm{~kb}$ )

Additional file 5: Gene ontology grouping of differentially-expressed genes. Representation of gene ontology grouping of 124 differentiallyexpressed genes into 44 different signaling pathways performed by Panther. (TIFF 1233 kb)

Additional file 6: Differentially-expressed genes in the same body structures as dusp6 and dusp2. The left column contains a list of all body structures of the zebrafish in which dusp6 and/or dusp2 are expressed, and the right column contains the identified differentially-expressed genes also expressed in those structures. (DOCX 14 kb)

Additional file 7: Additional patterning markers examined in dusp mutants. Wildtype (A, C, E, G, I, K, M, O, Q), dusp2/dusp6 double mutant (derived from crosses between dusp2 $2^{\text {um287/um287 }} ;$ dusp $^{\text {um286/um286 }}$ females and dusp2 $2^{\text {um287/um287 }}$;dusp $6^{\text {um286/um286 }}$ males; B, D, F, H, J, L, N), dusp2

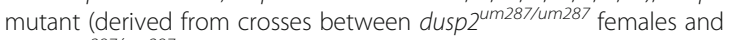
dusp2 $2^{\text {um287/um287 }}$ males; P) and dusp6 mutant (derived from crosses between dusp $6^{\text {um239/um239 }}$ females and dusp $6^{\text {um239/um239 }}$ males; R) embryos were analyzed by in situ hybridization for the expression of krox20, fgf3, fgf8, bmp2b, bmp4, chordin, and noggin1. (TIFF $3617 \mathrm{~kb}$ )

Additional file 8: Offspring from a single mutant parent have a milder phenotype. A. Comparison of the percent live embryos at $1 \mathrm{hpf}$ and $10 \mathrm{hpf}$ in clutches derived from various crosses between wildtype and homozygous mutant fish (specific genotypes are indicated below each bar). ANOVA+Dunnett's Multiple Comparison Test revealed a statistically significant decrease in live embryos at $10 \mathrm{hpf}$ for clutches where both parents are homozygous mutant for dusp6, but not when only one parent is homozygous mutant. A minimum of three clutches was 
analyzed for each cross with the mean percentages displayed \pm SD. B Comparison of the percent live embryos from $1 \mathrm{hpf}$ to $10 \mathrm{hpf}$ in clutches derived from crosses between wildtype and homozygous mutant fish (specific genotypes are indicated below the graph). A minimum of three clutches was analyzed for each cross with the mean percentages displayed \pm SD. (TIFF $2486 \mathrm{~kb})$

Additional file 9: DAPI staining detects embryos undergoing mitosis. Nuclear staining at approximately $1 \mathrm{hpf}$ shows that both wildtype and healthy dusp2/dusp6 double mutant (derived from crosses between

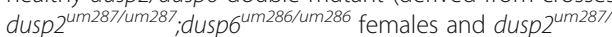
um287;dusp $6^{\text {um2 } 286 / u m 286}$ males) embryos can be detected at stages of mitosis when the chromatids are separated. (TIFF $841 \mathrm{~kb}$ )

\section{Abbreviations}

bp: Basepair; CAMP: Cyclic adenosine monophosphate; CRISPR: Clustered Regularly Interspaced Short Palindromic Repeats; DAPI: 4',6-diamidino-2phenylindole; Dusp: Dual specificity phosphatase; ERK: Extracellular signaling regulated kinase; Fgf: Fibroblast growth factor; FSH: Follicle stimulating hormone; hpf: Hours post fertilization; kb: Kilo basepair; LH: Luteinizing hormone; MAP: Mitogen activated protein; MEK: MAP/ERK kinase; MO: Morpholino oligonucleotide; NTP: Nucleoside tri-phosphate; PAM: Protospacer adjacent motif; PKA: Protein kinase A; qPCR: Quantitative polymerase chain reaction; r: Rhombomere; Spry: Sprout

\section{Acknowledgements}

We are grateful to Dr. Scot Wolfe for advice regarding CRISPR/Cas9 mutagenesis, to Dr. Nathan Lawson for providing the Cas9 plasmid, and to the Sagerström lab for helpful discussions and ideas. The following monoclonal antibodies were obtained from the Developmental Studies Hybridoma Bank, created by the $\mathrm{NICHD}$ of the $\mathrm{NIH}$ and maintained at The University of lowa, Department of Biology, lowa City, IA 52242: IsI1/2 (39.4D5) antibody developed by T. M. Jessell and S. Brenner-Morton; 3A10 antibody developed by T. M. Jessell, J. Dodd, and S. Brenner-Morton; and Zn8 antibody developed by B. Trevarrow. This work was supported by NIH grant NS038183 to CGS.

\section{Funding}

This work was supported by NIH grant NS038183 to CGS.

\section{Availability of data and materials}

The RNA-seq dataset generated and analyzed in the present study is available in the GEO repository at http://www.ncbi.n/m.nih.gov/geo/query/ acc.cgi?acc=GSE102793.

\section{Authors' contributions}

JMM participated in the design of the study, generated the germ line mutants, performed all phenotype analysis, and drafted the manuscript. CGS conceived the study, secured funding, participated in study design, and finalized the manuscript. Both authors read and approved the final manuscript.

\section{Ethics approval and consent to participate}

This study was submitted to and approved by the University of Massachusetts Medical School Institutional Animal Care and Use Committee and the University of Massachusetts Medical School Institutional Review Board.

\section{Consent for publication}

Not applicable.

\section{Competing interests}

The authors declare that they have no competing interests.

\section{Publisher's Note}

Springer Nature remains neutral with regard to jurisdictional claims in published maps and institutional affiliations.
Received: 17 August 2017 Accepted: 13 February 2018

Published online: 15 March 2018

\section{References}

1. Stulberg MJ, Lin A, Zhao H, Holley SA. Crosstalk between Fgf and Wnt signaling in the zebrafish tailbud. Dev Biol. 2012;369:298-307.

2. Ochi H, Ogino H, Kageyama Y, Yasuda K. The stability of the lens-specific Maf protein is regulated by fibroblast growth factor (FGF)/ERK signaling in lens fiber differentiation. J Biol Chem. 2003:278:537-44.

3. Aragon F, Pujades C. FGF signaling controls caudal hindbrain specification through Ras-ERK1/2 pathway. BMC Dev Biol. 2009:9:61.

4. Weisinger K, Kohl A, Kayam G, Monsonego-Ornan E, Sela-Donenfeld D. Expression of hindbrain boundary markers is regulated by FGF3. Biol Open. 2012;1:67-74

5. Walshe J, Maroon H, McGonnell IM, Dickson C, Mason I. Establishment of hindbrain segmental identity requires signaling by FGF3 and FGF8. Curr Biol. 2002;12:1117-23.

6. Weisinger K, Kayam G, Missulawin-Drillman T, Sela-Donenfeld D. Analysis of expression and function of FGF-MAPK signaling components in the hindbrain reveals a central role for FGF3 in the regulation of Krox20, mediated by Pea3. Dev Biol. 2010;344:881-95.

7. Thisse $B$, Thisse $C$. Functions and regulations of fibroblast growth factor signaling during embryonic development. Dev Biol. 2005;287:390-402.

8. Bermudez O, Pagès $\mathrm{G}$, Gimond $\mathrm{C}$. The dual-specificity MAP kinase phosphatases: critical roles in development and cancer. Am J Physiol Cell Physiol. 2010:299:C189-202.

9. Owens DM, Keyse SM. Differential regulation of MAP kinase signalling by dual-specificity protein phosphatases. Oncogene. 2007;26:3203-13.

10. Smith TG, Karlsson M, Lunn JS, Eblaghie MC, Keenan ID, Farrell ER, et al. Negative feedback predominates over cross-regulation to control ERK MAPK activity in response to FGF signalling in embryos. FEBS Lett. 2006;580:4242-5.

11. McGinnis W, Krumlauf R. Homeobox genes and axial patterning. Cell. 1992;68:283-302.

12. Amores A, Force A, Yan YL, Joly L, Amemiya C, Fritz A, et al. Zebrafish hox clusters and vertebrate genome evolution. Science. 1998;282:1711-4.

13. Choe S-K, Zhang X, Hirsch N, Straubhaar J, Sagerström CG. A screen for hoxb1-regulated genes identifies ppp1r14al as a regulator of the rhombomere 4 Fgf-signaling center. Dev Biol. 2011;358:356-67.

14. Chu Y, Solski PA, Khosravi-Far R, Der CJ, Kelly K. The mitogen-activated protein kinase phosphatases PAC1, MKP-1, and MKP-2 have unique substrate specificities and reduced activity in vivo toward the ERK2 sevenmaker mutation. J Biol Chem. 1996;271:6497-501.

15. Jeffrey KL, Brummer T, Rolph MS, Liu SM, Callejas NA, Grumont RJ, et al. Positive regulation of immune cell function and inflammatory responses by phosphatase PAC-1. Nat Immunol. 2006;7:274-83.

16. Zhang Q, Muller M, Chen CH, Zeng L, Farooq A, Zhou M-M. New insights into the catalytic activation of the MAPK phosphatase PAC-1 induced by its substrate MAPK ERK2 binding. J Mol Biol. 2005;354:777-88.

17. Rohan PJ, Davis P, Moskaluk CA, Kearns M, Krutzsch H, Siebenlist U, et al. PAC-1: a mitogen-induced nuclear protein tyrosine phosphatase. Science. 1993;259:1763-6.

18. Ward Y, Gupta S, Jensen P, Wartmann M, Davis RJ, Kelly K. Control of MAP kinase activation by the mitogen-induced threonine/tyrosine phosphatase PAC1. Nature. 1994:367:651-4.

19. Kim SC, Hahn JS, Min YH, Yoo NC, Ko YW, Lee WJ. Constitutive activation of extracellular signal-regulated kinase in human acute leukemias: combined role of activation of MEK, hyperexpression of extracellular signal-regulated kinase, and downregulation of a phosphatase, PAC1. Blood. 1999:93:3893-9.

20. Tsang M, Maegawa S, Kiang A, Habas R, Weinberg E, Dawid IB. A role for MKP3 in axial patterning of the zebrafish embryo. Development. 2004;131:2769-79.

21. Li C, Scott DA, Hatch E, Tian X, Mansour SL. Dusp6 (Mkp3) is a negative feedback regulator of FGF-stimulated ERK signaling during mouse development. Development. 2007;134:167-76.

22. Eblaghie MC, Lunn JS, Dickinson RJ, Münsterberg AE, Sanz-Ezquerro JJ, Farrell ER, et al. Negative feedback regulation of FGF signaling levels by Pyst1/MKP3 in chick embryos. Curr Biol. 2003:13:1009-18.

23. Kok FO, Shin M, Ni C-W, Gupta A, Grosse AS, van Impel A, et al. Reverse genetic screening reveals poor correlation between morpholino-induced and mutant phenotypes in zebrafish. Dev Cell. 2015;32:97-108. 
24. Rossi A, Kontarakis Z, Gerri C, Nolte H, Hölper S, Krüger M, et al. Genetic compensation induced by deleterious mutations but not gene knockdowns. Nature. 2015;524:230-3.

25. Kimmel CB, Ballard WW, Kimmel SR, Ullmann B, Schilling TF. Stages of embryonic development of the zebrafish. Dev Dyn. 1995;203:253-310.

26. Gagnon JA, Valen E, Thyme SB, Huang P, Ahkmetova L, Pauli A, et al. Efficient mutagenesis by Cas 9 protein-mediated oligonucleotide insertion and large-scale assessment of single-guide RNAs. PLoS One. 2014;9:e98186. Riley B, editor

27. Dolphin [Internet]. Available from: http://www.umassmed.edu/biocore/ introducing-dolphin/

28. Hauptmann G, Gerster T. Multicolor whole-mount in situ hybridization. Dev Biol Protoc. 2000;3:139-48. New Jersey: Humana Press

29. Zannino DA, Sagerström CG, Appel B. olig2-expressing hindbrain cells are required for migrating facial motor neurons. Dev Dyn. 2012;241:315-26.

30. Zhou B, Zhang J, Liu S, Reddy S, Wang F, Zhang Z-Y. Mapping ERK2-MKP3 binding interfaces by hydrogen/deuterium exchange mass spectrometry. $J$ Biol Chem. 2006;281:38834-44.

31. Keyse SM, Ginsburg M. Amino acid sequence similarity between CL100, a dual-specificity MAP kinase phosphatase and cdc25. Trends Biochem Sci. 1993;18:377-8.

32. Muda M, Boschert U, Smith A, Antonsson B, Gillieron C, Chabert C, et al. Molecular cloning and functional characterization of a novel mitogen-activated protein kinase phosphatase, MKP-4. J Biol Chem. 1997;272:5141-51.

33. ZFIN The Zebrafish Information Network [Internet]. Available from: http//_fin.org/. Accessed 18 July 2017

34. PANTHER - Gene List Analysis [Internet]. Available from: http://pantherdb.org/

35. Mi H, Thomas P. PANTHER pathway: an ontology-based pathway database coupled with data analysis tools. New York: Humana Press; 2009. p. 123-40.

36. Mi H, Huang X, Muruganujan A, Tang H, Mills C, Kang D, et al. PANTHER version 11: expanded annotation data from gene ontology and Reactome pathways, and data analysis tool enhancements. Nucleic Acids Res. 2017;45: D183-9. Oxford University Press

37. ZFIN Downloads Archive [Internet]. Available from: http://zin.org/downloads. Accessed 18 July 2017.

38. Kondoh K, Torii S, Nishida E. Control of MAP kinase signaling to the nucleus. Chromosoma. 2005;114:86-91.

39. Lemmon MA, Freed DM, Schlessinger J, Kiyatkin A. The dark side of cell signaling: positive roles for negative regulators. Cell. 2016;164:1172-84.

40. Shilo B-Z. The regulation and functions of MAPK pathways in drosophila. Methods. 2014;68:151-9.

41. Niehrs C, Meinhardt H. Modular feedback. Nature. 2002;417:35-6.

42. Evans DRH, Hemmings BA. Signal transduction. What goes up must come down. Nature. 1998;394:23-4

43. Hendzel MJ, Wei Y, Mancini MA, Van Hooser A, Ranalli T, Brinkley BR, et al. Mitosis-specific phosphorylation of histone $\mathrm{H} 3$ initiates primarily within pericentromeric heterochromatin during G2 and spreads in an ordered fashion coincident with mitotic chromosome condensation. Chromosoma. 1997;106:348-60.

44. Budirahardja Y, Gönczy P. Coupling the cell cycle to development. Development. 2009;136:2861-72.

45. Lee MT, Bonneau AR, Takacs CM, Bazzini AA, DiVito KR, Fleming ES, et al. Nanog, Pou5f1 and SoxB1 activate zygotic gene expression during the maternal-to-zygotic transition. Nature. 2013;503:360-4.

46. González-Fernández L, Ortega-Ferrusola C, Macias-Garcia B, Salido GM, Peña FJ, Tapia JA. Identification of protein tyrosine phosphatases and dual-specificity phosphatases in mammalian spermatozoa and their role in sperm motility and protein tyrosine phosphorylation. Biol Reprod. 2009;80:1239-52.

47. Spencer SL, Gaudet S, Albeck JG, Burke JM, Sorger PK. Non-genetic origins of cell-to-cell variability in TRAIL-induced apoptosis. Nature. 2009;459:428-32.

48. Feinerman O, Veiga J, Dorfman JR, Germain RN, Altan-Bonnet G. Variability and robustness in $\mathrm{T}$ cell activation from regulated heterogeneity in protein levels. Science. 2008;321:1081-4.

49. Ferrell JE. Tripping the switch fantastic: how a protein kinase cascade can convert graded inputs into switch-like outputs. Trends Biochem Sci. 1996;21:460-6.

50. Cheong R, Rhee A, Wang CJ, Nemenman I, Levchenko A. Information transduction capacity of noisy biochemical signaling networks. Sci. 2011;334:354-8

51. Jeschke M, Baumgartner S, Legewie S. Determinants of cell-to-cell variability in protein kinase signaling. PLoS Comput Biol. 2013;9:e1003357.
52. Fürthauer $M$, Lin W, Ang S-L, Thisse B, Thisse C. Sef is a feedbackinduced antagonist of Ras/MAPK-mediated FGF signalling. Nat Cell Biol. 2002;4:170-4.

53. Tsang M, Friesel $R$, Kudoh $T$, Dawid IB. Identification of Sef, a novel modulator of FGF signalling. Nat Cell Biol. 2002;4:165-9.

54. Nagahama Y, Yamashita M. Regulation of oocyte maturation in fish. Develop Growth Differ. 2008;50:S195-219.

55. Donaubauer EM, Law NC, Hunzicker-Dunn ME. Follicle-stimulating hormone (FSH)-dependent regulation of extracellular regulated kinase (ERK) phosphorylation by the mitogen-activated protein (MAP) kinase phosphatase MKP3. J Biol Chem. 2016;291:19701-12.

56. Sen A, Caiazza F. Oocyte maturation: a story of arrest and release. Front Biosci. 2013;5:451-77.

57. Su Y-Q, Denegre JM, Wigglesworth K, Pendola FL, O'Brien MJ, Eppig JJ. Oocyte-dependent activation of mitogen-activated protein kinase (ERK1/2) in cumulus cells is required for the maturation of the mouse oocytecumulus cell complex. Dev Biol. 2003;263:126-38.

58. Prochazka R, Blaha M. Regulation of mitogen-activated protein kinase $3 / 1$ activity during meiosis resumption in mammals. J Reprod Dev. 2015;61:495-502.

59. Sette C, Barchi M, Bianchini A, Conti M, Rossi P, Geremia R. Activation of the mitogen-activated protein kinase ERK1 during meiotic progression of mouse pachytene spermatocytes. J Biol Chem. 1999;274:33571-9.

60. Di Agostino S, Rossi P, Geremia R, Sette C. The MAPK pathway triggers activation of Nek2 during chromosome condensation in mouse spermatocytes. Development. 2002;129:1715-27.

61. Di Agostino S, Fedele M, Chieffi P, Fusco A, Rossi P, Geremia R, et al. Phosphorylation of high-mobility group protein A2 by Nek2 kinase during the first meiotic division in mouse spermatocytes. Mol Biol Cell. 2004;15:1224-32.

62. Griswold MD. The central role of Sertoli cells in spermatogenesis. Semin Cell Dev Biol. 1998;9:411-6.

63. Cheng J, Watkins SC, Walker WH. Testosterone activates mitogen-activated protein kinase via Src kinase and the epidermal growth factor receptor in Sertoli cells. Endocrinology. 2007;148:2066-74.

64. Fan H-Y, Shimada M, Liu Z, Cahill N, Noma N, Wu Y, et al. Selective expression of KrasG12D in granulosa cells of the mouse ovary causes defects in follicle development and ovulation. Development. 2008:135:2127-37.

65. Fan H-Y, Liu Z, Shimada M, Sterneck E, Johnson PF, Hedrick SM, et al. MAPK3/1 (ERK1/2) in ovarian granulosa cells are essential for female fertility. Science. 2009;324:938-41.

66. Miraoui H, Dwyer AA, Sykiotis GP, Plummer L, Chung W, Feng B, et al. Mutations in FGF17, IL17RD, DUSP6, SPRY4, and FLRT3 are identified in individuals with congenital hypogonadotropic hypogonadism. Am J Hum Genet. 2013;92:725-43.

67. Clift D, Schuh M. Restarting life: fertilization and the transition from meiosis to mitosis. Nat Rev Mol Cell Biol. 2013;14:549-62.

68. Taylor SM, Nevis KR, Park HL, Rogers GC, Rogers SL, Cook JG, et al. Angiogenic factor signaling regulates centrosome duplication in endothelial cells of developing blood vessels. Blood. 2010;116:3108-17.

69. Yun C, Cho H, Kim S-J, Lee J-H, Park SY, Chan GK, et al. Mitotic aberration coupled with centrosome amplification is induced by hepatitis B virus X oncoprotein via the Ras-mitogen-activated protein/ extracellular signal-regulated kinase-mitogen-activated protein pathway. Mol Cancer Res. 2004;2:159-69.

70. Colello D, Mathew S, Ward R, Pumiglia K, LaFlamme SE. Integrins regulate microtubule nucleating activity of centrosome through mitogen-activated protein kinase/extracellular signal-regulated kinase kinase/extracellular signal-regulated kinase (MEK/ERK) signaling. J Biol Chem. 2012;287:2520-30. 